pessimism of doctors regarding the prognosis of pregnancy in SLE left many patients feeling confused and depressed. Changes in physical appearance such alopecia, rashes and weight fluctuations were also a major concern affecting self-image and libido, often leading to strained relationships and breakups. Coping mechanisms included intense spiritual beliefs giving them the courage to 'push through the difficult times'. Use of alternative therapies was common, which they believed helped contain the symptoms of SLE. These included drinking 'blessed' water, traditional herbal remedies, and soothing leaf or pressure applications to painful joints. A poor understanding of SLE by the patients themselves, family and the community, coupled with the unpredictable course of the disease, exacerbated frustration and uncertainty. For many patients, limited income, lack of basic services like public transport, dependency of family members, and comorbid diseases, such as HIV and tuberculosis, exacerbated the negative daily experiences of living with SLE. Patients felt that patient-led support groups, better education by health professionals, and public awareness of SLE would help them cope with the disease.

Conclusions: Indigent South African women with SLE in SA have complex, chronic and challenging life experiences. A poor understanding and acceptance of SLE by patients themselves, compounded by a background of poverty and a perception of being misunderstood by family members, health professionals and the community at large had negative impact on multiple dimensions of patient's lives.

Acknowledgements: All women and funding from Thuthuka grant and the Harry Crossley Foundation.

Disclosure of Interest: None declared

DOI: 10.1136/annrheumdis-2018-eular.7254

\section{AB1443-HPR EXPLORING THE RELATION BETWEEN IMPAIRMENT RATING BY DAS-28 AND BODY FUNCTION, ACTIVITY- PARTICIPATION AND ENVIRONMENTAL FACTORS BASED ON ICF IN THE PATIENT WITH RHEUMATOID ARTHRITIS}

E. Gur Kabull ${ }^{1}$ U. Bas Aslan ${ }^{1}$, B Basakci Calik ${ }^{1}$, M. Tasci' ${ }^{2}$, V. Cobankara ${ }^{2} .{ }^{1}$ School of Physical Therapy and Rehabilitation, Pamukkale University; ${ }^{2}$ Department of Internal Medicine, Division of Rheumatology, Pamukkale University School of Medicine, Denizli, Turkey

Background: Rheumatoid Arthritis (RA) is the cause of functional loss and disability of individuals. It also results in negative effects on the emotional components as well as physical components related to health. If specific domains of medical condition criteria are systematically linked with relevant parts of ICF, we will have a common conceptual understanding of ICF and patient-oriented health criteria. It may also make clinical practices easy.

Objectives: The aim of this study was to link and allocate items of disability questionnaires with body function, activity participation and environmental factors based on ICF Hand Core Set. The other objective of this study is to examine the relationship between the impairment and the ICF components determined on the basis of disability questionnaires in participants with Rheumatoid Arthritis.

Methods: Impairment was evaluated by use of DAS28 and disability by Disabilities of Arm, Shoulder and Hand Questionnaire (DASH), Michigan Hand Outcomes Questionnaire (MHQS), Duruöz Hand Index (DHI), Arthritis Impact Measurement Scales 2 (AIMS 2) in participants with rheumatoid arthritis $(n=100)$. Items of DASH, MHQS, DHI and AIMS 2 were linked and allocated with the ICF Hand Core Set as a result of three expert opinions. The data was analysed using Kappa index and Pearson's correlation coefficient.

Results: Based on expert distinction on DASH, MHQS, DEI and AIMS 2 items, MHQS covered the highest number of body function categories of ICF Hand Core Set with 8 and AIMS 2 covered the highest number of ICF Hand Core Set with totally 25 (body function=3, activity participation $=20$, environmental factors $=2$ ). While impairment (DAS28) had moderate correlation with subjective impairment (body function scores considered as subjective impairment) and activity participation for all assessment questionnaires $(r=0.50-0.69)$; subjective impairment had high correlation with activity participation for DASH and MHQS ( $r>0.70)$ and also had moderate correlation with activity participation for AIMS 2 $(r=0.50-0.69)$. Furthermore, subjective impairment had weak correlation with environmental factors $(r=0.10-0.49)$.

Conclusions: We suggest that AIMS 2 is the most appropriate for clinicians and researchers who aim to perform a more comprehensive biopsychosocial assessment in patients with RA. In addition, the clinician's assessments and the impairment levels reported by the patients are interrelated and the impairment levels reported by patients is also affected by environmental factors.

\section{REFERENCES:}

[1] Somers, T, Shelby R, Keefe F, Godiwala B, Lumley M, Mosley-Williams A, et al. Disease severity and domain specific arthritis self-efficacy: relationships to pain and functioning in Rheumatoid Arthritis. Arthritis Care Res (Hobroken) 2010; 62(6): 848-856.

[2] Corbacho M, Dapueto J. Assesing the functional status and quality of life of patients with Rheumatoid Arthritis. Brazilian Journal of Rheumatology 2010; 50(1): 31-43.

[3] Cieza A, Brockow T, Ewert T, Amman E, Kollerits B, Chatterji S, et al. Linking health-status measurements to the international classification of functioning, disability and health. J Rehabil Med 2002; 34(5): 205-210.

Disclosure of Interest: None declared

DOI: 10.1136/annrheumdis-2018-eular.6189

\section{AB1444-HPR UNDERSTANDING FATIGUE BURDEN AND COPING STRATEGIES IN RHEUMATOID ARTHRITIS USING QUALITATIVE RESEARCH}

C Zhang ${ }^{1}$, Y. Shaw ${ }^{2}$, B. Shakley ${ }^{1}$, L. Ferri ${ }^{3}$, K. Michaud ${ }^{2}$, D. McDonald ${ }^{1}$, T. A. Simon ${ }^{3} .{ }^{1}$ LIFT1428, Chattanooga; ${ }^{2}$ National Data Bank for Rheumatic Diseases, Wichita; ${ }^{3}$ Bristol-Myers Squibb, Princeton, USA

Background: Fatigue is a major symptom of RA but is under-represented when considering an appropriate treatment and in the measurement of disease activity. At the same time, patients' perspectives on fatigue in the overall context of their RA and life have not been studied in detail before. To understand that perspective, we aimed to explore patients lived experience with RA-related fatigue.

Objectives: We explored the narratives of patients with RA and their discourse on RA-related fatigue to assess the burden of disease and coping strategies.

Methods: Semi-structured interviews were conducted with adult patients with RA living in two geographical regions in the United States. Interviewees were asked to discuss their experiences with diagnosis, symptoms, physical and social limitations, coping with challenges, treatment and healthcare providers. The interviews were audio-recorded and transcribed verbatim. Transcripts were analysed for themes related to the burden of RA-related fatigue and coping strategies.

Results: Eighteen patients were interviewed, with ages ranging from 27 to 80 years and RA duration ranging from 4 to 40 years. Four themes emerged around the impact of RA-related fatigue, fatigue in context of RA symptoms, coping strategies and coping success (table 1).

Abstract AB1444HPR - Table 1. Burden of RA-Related Fatigue and Coping Strategies Themes

Theme Details

Fatigue limits RA patients' ability to - Patients with RA have limited energy reserves to live their lives perform common activities of daily living Fatigue creates 'brain fog' that reduces RA patients' ability to focus and diminishes academic and job performance

Patients with RA adjust their future career choices based on these limitations

Fatigue and pain are intertwined

- Fatigue is strongly connected with pain and it is difficult for patients with RA to separate the two

symptoms. Pain and active RA symptoms increase fatigue. Fatigue also amplifies pain

Fatigue can be a bigger concern than pain.

Patients with RA find it harder to manage fatigue compared with pain

Coping with fatigue is an internal struggle

- Patients with RA are faced with the tough choice of giving in to the fatigue or pushing through and risk exceeding energy reserves

Patients with RA who have difficulty coping with

fatigue feel frustrated, embarrassed and inadequate

- Patients with RA use a variety of coping strategies, often in combination

Acceptance of limitations leads to more effective coping strategies

Coping strategies include pushing through the

fatigue, using distractions, pacing oneself, sleeping, drinking coffee and using medication

Patients with RA who accept their limitations are

more likely to pace themselves, as opposed to

pushing through their limits, and have fewer

negative emotions when they give up

Conclusions: Fatigue is a major concern for patients with RA and can be more debilitating than pain. A patient's constant battle with fatigue can have physical, mental and emotional consequences. 